\title{
Optimizing Vitamin D Status Improves Outcomes in Critical Ill and Injured Patients
}

\author{
Omar K Danner ${ }^{1 *}$, Erin Danielle Danner ${ }^{2}$ and Leslie Ray Matthews ${ }^{1}$ \\ ${ }^{1}$ Department of Surgery, Morehouse School of Medicine, USA \\ ${ }^{2}$ College of Arts and Sciences, University of Georgia, USA
}

*Corresponding author: Omar K Danner, Department of Surgery, Morehouse School of Medicine, USA.

To Cite This Article: Omar K Danner, Optimizing Vitamin D Status Improves Outcomes in Critical Ill and Injured Patients. 2020 - 8(2). AJBSR. MS.ID.001250. DOI: 10.34297/AJBSR.2020.08.001250.

Received: 眥 March 03, 2020; Published: 些 March 16, 2020

Abbreviations: ICU: Intensive Care Unit; BMD: Maximal Bone Density; VDR: Vitamin D Receptors; ARDS: Acute Respiratory Distress Syndrome

\section{Introduction}

In 2008, Giovanucci et al. [1] showed that men with low vitamin D levels suffered 2.42 times more myocardial infarctions than those with normal vitamin D status [1]. Alternatively, a sufficient amount of serum 25-hydroxyvitamin D3, [25(OH)D], appears to improve the risk of almost every disease of aging. Dobnig et al. [2] similarly demonstrated people with an inadequate vitamin D status have twice the likelihood of death over seven years [2,3]. Vitamin D sufficiency, define as serum 25(OH)D levels of 30ng/ $\mathrm{mL}(75 \mathrm{nmol} / \mathrm{L})$ and above, improves various health outcomes, including bone mineral density, fractures, and colorectal cancer, based on analysis of observational studies [4]. Vitamin D levels of 21-29ng/mL delineates insufficiency and a concentration of 20ng/ $\mathrm{mL}$ or less defines vitamin D deficiency [4]. When vitamin D levels are inadequate, and particularly fall below $17.8 \mathrm{ng} / \mathrm{mL}$, mortality risk increases by as much as $26 \%$ from all-cause mortality in the general population $[3,5]$. Current data suggests hypovitaminosis D plays a significant role in the development of numerous common chronic diseases and inflammatory conditions, including coronary artery disease, cerebrovascular disease, diabetes mellitus, autoimmune disorders, such as systemic lupus erythematosus, scleroderma and multiple sclerosis and 17 different forms of cancer [6-12]. Vitamin D deficiency is arguably one of the most prevalent but underrated nutritional deficiency worldwide [6,7]. It continues to be associated with increased overall mortality risk in several studies [8-12]. Several observational studies on hypovitaminosis D over the past decade support the persistent existence of a high prevalence of vitamin D insufficiency and deficiency in as many as 50 to $90 \%$ of hospitalized patients, with a particular predilection in the setting of critical illness [6-9, 12-14]. Although full elucidation of the role vitamin D status plays in patients requiring admission to the intensive care unit (ICU) is an area undergoing active investigation, epidemiological data supports the findings that vitamin D sufficiency may decrease the risk of systemic inflammatory from all causes as well as sepsis in general $[12,15]$. Investigations by our group examining the prevalence and effects of insufficient or deficient serum vitamin D levels on our critically-ill and/or severelyinjured trauma patients revealed increased risk of unfavorable outcomes when serum vitamin D levels fell below a level of 30ng/ $\mathrm{mL}(75 \mathrm{nmol} / \mathrm{mL}]$ in SICU trauma patients who survived their initial injury and resuscitative efforts $[6,16]$. According to some of the world's leading vitamin D experts, optimal serum levels of 25[OH]D range between 30 and 50ng/mL. Those individuals with $25(\mathrm{OH}) \mathrm{D} 3$ (calcidiol) levels below $30 \mathrm{ng} / \mathrm{mL}$ are considered to have vitamin D insufficiency as noted above $[5,6,8,15,16]$ looked at the risk of 30 day and in-hospital mortality after initiation of critical care services in patients with severe vitamin D deficiency. There was a 1.9-fold higher risk of death than in those patients with vitamin D levels of $\leq 30 \mathrm{ng} / \mathrm{mL}$ [7]. Inadequate levels of 25 [OH]D in the insufficient cohort remained a significant predictor of increased likelihood of mortality, even after multivariate adjustment [7].

Under normal circumstances, vitamin D is a hormone produced principally by the skin in response to natural sunlight. However, 
when the sun hits the northern hemisphere below 45 degrees in the spring and summer, it cannot produce UVB rays of the proper wavelengths from 290 to $315 \mathrm{~nm}$, which is required for the production of vitamin D in the skin between 10:00 a.m. and approximately 3:00 p.m. [6,17]. At latitudes of 32 degrees or above, vitamin D levels are the lowest, particularly during the winter months and early spring, due to the lack of sunlight at the suitable wavelength $[6,17]$. During the fall and winter months, vitamin D stores decrease by approximately 20 to 30\%. Furthermore, vitamin D deficiency is very common in Western society as it is nearly impossible to get adequate amounts of vitamin $\mathrm{D}$ from diet alone without purposeful exogenous supplementation $[6,7,17]$. Therefore, reversal of hypovitaminosis D may not be quick or easy without supplementation.

There is a suggestion by researchers that vitamin D deficiency is present in at least $50 \%$ to $80 \%$ of critically ill patients admitted to surgical and medical ICUs. Nevertheless, based on the definition of normal and subtherapeutic vitamin D status, vitamin D insufficiency in western society may be grossly underestimated [6-14,16]. The true prevalence and extent of vitamin D deficiency might be much worse. This understanding is particularly pertinent considering we have entered the season of increased risk for acute respiratory illness, where a robust immune system is of paramount importance for fighting off upper respiratory tract infections. This underappreciation of vitamin D deficiency may be associated with increased relative risk of adverse outcomes in fragile and critically ill patient populations [6-14,16]. In our prior investigations, we evaluated a cutoff $\geq 40 \mathrm{ng} / \mathrm{mL}$ as a surrogate marker to define a normal vitamin D level, and levels of $<40 \mathrm{ng} / \mathrm{mL}$ to represent a relative insufficiency in our ICU patients [6]. We found higher 25[OH]D levels improved outcomes in surgical ICU patients $[6,8,16]$. There is other evidence that supports the body functions better at this higher vitamin D level. Other authors have suggested that maximal bone density (BMD) can only be achieved when the 25-hydroxyvitamin D level reaches $40 \mathrm{ng} / \mathrm{mL}$ or greater [17]. As fractures are very common in critically injured elderly trauma patients, achieving adequate vitamin D levels becomes increasingly important in this fragile, at-risk patient population. Based on prior observations demonstrating the need for calcidiol levels $\geq 40 \mathrm{ng} /$ $\mathrm{mL}$ to adequately suppress serum parathyroid hormone levels and achieve maximal bone density in the hips and lumbar spine patients, it is plausible to suggest that higher vitamin D levels may portend a protective effect in the other severely injured and/or critically ill individuals [5,6,15,18-20].

To achieve a vitamin D of 30 to $50 \mathrm{ng} / \mathrm{mL}$ and maintain it long term, investigators have found that it takes over 4,000 to 5,000 units of $25-(0 \mathrm{H})$ vitamin D supplementation per day [18,20]. This amounts to over 5 to 10 times the current recommended daily intake [20]. The importance of this recommendation is that optimal local concentrations of serum $25-(\mathrm{OH}) \mathrm{D} \geq 30 \mathrm{ng} / \mathrm{mL}[6,24,25]$ are required for optimal paracrine conversion to 1,25-(OH)2-vitamin D (calcitriol) by macrophages and other immune cells. The serum concentration needs to be $\geq 30$ (>75 nmol/L) [6,7,21-24] to activate the vitamin D receptors (VDR) that regulates the immune response. Activation of the VDR by bioactive vitamin D up-regulates the anti-inflammatory cytokines IL-8 and IL-10, which promotes the expression of a T-suppressor cell lineage and helps to turn off the adaptive immune response once the job is complete [23-25]. Thus, it is understandable that patients with moderate to severe vitamin D deficiency syndromes are less capable of mounting a successful immune response to severe insults, injury, and acute viral and bacterial infections. Furthermore, they have higher risk of ICUrelated septic complications, acute respiratory distress syndrome (ARDS) and death.

\section{Conclusion}

Published data suggests critically-ill patients with inadequate serum vitamin D stores may enter into a vicious inflammatory cycle due to low levels of the bioactive form of vitamin D, calcitriol, which leads to increased production of pro-inflammatory cytokines, which may not be easily reversed with vitamin D supplementation $[6,8,24]$. This review may provide a plausible link between the excess mortality observed in people during an acute outbreak of novel acute respiratory syndromes in the general population and especially patients in the ICU setting who are at a higher risk of frankly deficient vitamin D levels. Therefore, we recommend checking serum 25-OH vitamin D levels on all hospitalized ICU patients, and particularly those with acute respiratory or unexplained infectious/inflammatory illness. Although it is difficult to prove clinical effectiveness in the setting of acute infectious illness, supplementation of vitamin D stores to augment the immune system using cholecalciferol (Vitamin D3) at doses of 4,000 to 5,000 units daily may not be unreasonable, especially during this uncharacteristically aggressive flu season, and more so in light of the worldwide coronavirus [COVID-19] epidemic.

\section{References}

1. Giovannucci E, Liu Y, Hollis BW, Rimm EB (2008) 25-hydroxyvitamin $\mathrm{D}$ and risk of myocardial infarction in men: a prospective study. Arch Intern Med 168(11): 1174-1180.

2. Dobnig H, Pilz S, Scharnagi H, Renner W, Seelhorst U, et al. (2008) Independent association of low serum 25-hydroxyvitamin $\mathrm{d}$ and 1,25-dihydroxyvitamin D levels with all-cause and cardiovascular mortality, Arch Intern Med 168(12): 1340-1349.

3. Melamed ML, Michos ED, Post W, Astor B (2008) 25-hydroxyl vitamin D levels and the risk of mortality in the general population, Arch Intern Med 168(15): 1629-1637.

4. Bischoff-Ferrari HA, Giovannucci E, Willett WC, Dietrich T, DawsonHughes B, et al. (2006) Estimation of optimal serum concentrations of 25-hydroxyvitamin D for multiple health outcomes. Am J Clin Nutr 84(1): 18-28.

5. Amer M, Qayyam R (2013) Relationship between 25-Hydroxyvitamin D and All-Cause and Cardiovascular Disease Mortality. Am J Med 126(6): 509-514. 
6. Matthews LR, Ahmed Y, Dennis-Griggs D, Wilson K, Danner OK, et al (2012) Worsening severity of vitamin D deficiency is associated with increased length of stay, surgical intensive care unit cost, and mortality rate in surgical intensive care unit patients. Am J Surg 204(1):37-43.

7. Holick MF (2007) Vitamin D deficiency. N Engl J Med 357(3): 266-281.

8. Braun A, Chang D, Mahadevappa K, Gibbons FK, Liu Y, et al. (2011) Association of low serum 25-hydroxyvitamin D levels and mortality in the critically ill. Critical Care Medicine 39(4): 671-677.

9. Flynn L, Zimmerman LH, McNorton K, et al. (2012) Effects of vitamin D deficiency in critically ill surgical patients. American Journal of Surgery; 203(3): 379-382.

10. Venkatram S, Chilimuri S, Adrish M, Salako A, Patel M, et al. (2011) Vitamin D deficiency is associated with mortality in the medical intensive care unit. Critical Care 15(6): 292.

11. Lee P, Eisman JA, Center JR (2009) Vitamin D deficiency in the intensive care unit: an invisible accomplice to morbidity and mortality, Intensive Care Med 35(12): 2028-2032.

12. Lee P, Eisman JA, Center JR (2009) Vitamin D deficiency in critically ill patients, N Engl J Med 360(18): 1912-1914.

13. Arnson Y, Gringauz I, Itzhaky D, Amittal H (2012)Vitamin D deficiency is associated with poor outcomes and increased mortality in severely ill patients, OJM: An International Journal of Medicine 105(7): 633-639.

14. Lucidarme O, Messai E, Mazzoni T, Arcade M, du Cheyron D (2010) Incidence and risk factors of vitamin D deficiency in critically ill patients: results from a prospective observational study, Intensive Care Med 36(9):1609-1611.

15. Grant W (2009) Solar ultraviolet-B irradiance and vitamin D may reduce the risk of septicemia, Dermatoendocrinol 1(1): 37-42.

16. Danner OK, Ruben Burbank, Diane Dennis-Griggs, Kenneth L Wilson, Frank Jones, et al. (2016) Vitamin D Insufficiency is Associated with a Higher Risk of In-Hospital Mortality in Critically-Injured Trauma Patients. EC Nutrition 4(6): 996-1005.
17. Binkley N, Novotny R, Krueger D, Kawahara T, Daida YG, (2007) Low Vitamin D Status despite Abundant Sun Exposure. The Journal of Clinical Endocrinology \& Metabolism 92(6): 2130-2135.

18. Vieth R (1999) Vitamin D supplementation, 25-hydroxyvitamin D concentrations, and safety. Am J Clin Nutr 69(5): 842-856.

19. Thacher TD, Clarke BL (2011) Vitamin D insufficiency. Mayo Clin Proc. 86(1): 50-60.

20. Dawson-Hughes B, Heaney RP, Holick MF, Lips P, Meunier PJ, et al. (2005) Estimates of optimal vitamin D status, Osteoporos Int 16(7): 713-716.

21. Kinyamu HK, Gallagher JC, Rafferty KA, Balhorn KE (1998) Dietary calcium and vitamin D intake in elderly women: effect on serum parathyroid hormone and vitamin D metabolites. Am J Clin Nutr 67(2): 342-348.

22. Hollis BW (2004) The determination of circulating 25-hydroxyvitamin D: no easy task. J Clin Endocrinol Metab 89(7): 3149-3151.

23. Hollis BW, Wagner CL (2011) Vitamin D requirements and supplementation during pregnancy. Current Opinion in Endocrinology, Diabetes and Obesity 18(6): 371-375.

24. MathieuC, L Adorini (2002) The coming of age of 1,25-dihydroxyvitamin D-3 analogs as immunomodulatory agents. Trends Mol Med 8(4): 174 179.

25. Danner OK, Matthews LR, Francis S, Rao VN, Harvey CP, et al. (2016) Vitamin D3 suppresses class II invariant chain peptide expression on activated B-lymphocytes: a plausible mechanism for downregulation of acute inflammatory conditions. Journal of Nutrition and Metabolism pp.1-9. 